\title{
Lercanidipine/enalapril combination in the management of obesity-related hypertension
}

This article was published in the following Dove Press journal:

Integrated Blood Pressure Control

26 April 2016

Number of times this article has been viewed

\author{
Guido Grassi ${ }^{1,2}$ \\ 'Internal Medicine, Department of \\ Health Science, Università Milano- \\ Bicocca, Milano, Italy; ${ }^{2}$ Istituto di \\ Ricerche a Carattere Scientifico \\ (IRCCS) Multimedica, Sesto San \\ Giovanni, Milano, Italy
}

\begin{abstract}
Obesity-related hypertension represents a condition frequently observed in current clinical practice characterized by a complex pathophysiological background and a very high cardiovascular risk profile, particularly in severely obese individuals. This explains, on the one hand, the difficulty in reducing elevated blood pressure values in this pathological state and, on the other, the need to achieve this goal in a relatively short-time period to prevent the occurrence of fatal and nonfatal cardiovascular events. Both nonpharmacological and pharmacological measures are available in the therapeutic approach for this condition. Among the pharmacological interventions, a combination of two antihypertensive drugs represents the most common recommended strategy aimed at achieving blood pressure control. This paper, after briefly examining the main pathophysiological features of obesity-related hypertension, will review the importance in the treatment of this condition of the drug combination based on a calcium channel blocker and an angiotensin-converting enzyme inhibitor, with specific focus on lercanidipine/enalapril. Following an analysis of the main pharmacological properties of the combination, the results of the studies based on this pharmacological approach in obesity-related hypertension will be critically discussed. The efficacy, safety, and tolerability profile of the lercanidine/enalapril drug combination as well as its potential limitations will also be examined.
\end{abstract}

Keywords: obesity-related hypertension, antihypertensive drug treatment, combination drug, lercanidipine/enalapril

\section{Introduction}

The term "obesity-related hypertension" refers to the clinical condition frequently detected in daily practice characterized by the presence of clinic blood pressure values above $140 / 90 \mathrm{mmHg}$ in patients with a body mass index greater than $30 \mathrm{~kg} / \mathrm{m}^{2}$. There are several pathophysiological and clinical features of this pathological association. They include, for example, the finding that the activation of different neurohumoral systems involved in cardiovascular homeostasis control represents one of the major pathophysiological mechanisms responsible for the blood pressure elevation. ${ }^{1}$ The process also includes a marked stimulation of the adrenergic nervous system, the renin-angiotensin-aldosterone axis, the atrial natriuretic peptides and the nitric oxide network, as well as activation of the vascular inflammatory process. ${ }^{1,2}$ Among the clinical features of the disease, the metabolic abnormalities affecting lipids, triglycerides, glycemic, and insulin profile should also be included. The association of these alterations combined with visceral (or central) obesity and hypertension leads to the occurrence of the so-called metabolic syndrome. ${ }^{3}$ A final feature of obesity-related hypertension is represented by the evidence that it carries a very high cardiovascular
Internal Medicine, Department of Health

Science, Università Milano Bicocca,

Ospedale San Gerardo dei Tintori, Via Pergolesi 33, 20052 Monza, Milano, Italy

Tel +390392333 6001

Email guido.grassi@unimib.it
Integrated Blood Pressure Control 2016:9 69-77

(c) (1) (8) ๑ 2016 Grassi. This work is published and licensed by Dove Medical Press Limited. The full terms of this license are available at https://www.dorepress.com/terms.php and (c) 1 BY 1 incorporate the Creative Commons Attribution - Non Commercial (unported, v3.0) License (http:///creativecommons.org/licenses/by-nc/3.0/). By accessing the work you hereby accept the Terms. Non-commercial uses of the work are permitted without any further permission from Dove Medical Press Limited, provided the work is properly attributed. For permission
for commercial use of this work, please see paragraphs 4.2 and 5 of our Terms (https://www.dovepress.com/terms.php).
Dovepress

http://dx.doi.org/10.2147/1BPC.592779

\section{9}

d


risk, the concomitant presence of abnormally increased body fat and elevated blood pressure values exerting additive adverse effects on cardiovascular prognosis. ${ }^{4,5}$ In obese hypertensive patients, this takes place because of the occurrence of major cardiovascular complications (such as congestive heart failure, renal failure, obstructive sleep apnea, cardiac arrhythmias, and sudden death), major metabolic alterations (diabetes mellitus, dyslipidemia, and insulin resistance), and the frequent development of end-organ damage (left ventricular hypertrophy, left ventricular diastolic dysfunction, carotid plaques, microalbuminuria, vascular atherosclerosis, and early vascular aging).,5

The complex pathophysiological background of obesityrelated hypertension combined with its very high cardiovascular risk profile may explain, as outlined by current guidelines and recommendations issued by international scientific societies, not only the clinical difficulties but also the clinical relevance of controlling blood pressure values in these patients. ${ }^{4-6}$ This paper will focus on the experience accumulated in the past few years with the use of the antihypertensive drug combination treatment lercanidipine/ enalapril in the management of obesity-related hypertension. The rationale for this choice includes 1) the fact that the combination examined is based on an angiotensin-converting enzyme (ACE) inhibitor and a calcium antagonist, and thus it is one of the therapeutic approaches recommended by guidelines in the treatment of obesity-related hypertension ${ }^{4-6}$ and 2) De Ciuceis et $\mathrm{al}^{7}$ have evaluated this combination in different studies, with particular focus on its metabolic and blood pressure lowering effects in obese hypertensives. Specifically, the paper will discuss in sequence 1) the blood pressure lowering therapeutic options in the treatment of high blood pressure in obese hypertensive patients, 2) the pharmacological profile of the drug combination lercanidipine/ enalapril, 3) its antihypertensive and metabolic effects, 4) its safety and tolerability profile, and finally 5) its impact in terms of patient's focused outcomes, with particular emphasis on compliance to treatment as well as on cardiovascular risk protection.

\section{Blood pressure management options in obesity-related hypertension}

Both international guidelines and recommendations issued by scientific societies, such as those jointly published by the European Society of Hypertension and the European Association for the Study of Obesity, emphasize the importance of cardiovascular risk reduction of blood pressure lowering interventions in obesity-related hypertension. ${ }^{4-6}$ They also recognize the practical difficulties in achieving such goals, given the evidence that in this clinical condition 1) the mechanisms leading to the blood pressure elevation are much more multifactorial than the ones reported for the essential hypertensive state, not complicated by overweight obese state, ${ }^{1,2,5,6} 2$ ) the frequent association of hypertension with the metabolic, neurohumoral, and cardiovascular alterations (including obstructive sleep apnea) mentioned earlier may represent a factor favoring the occurrence of a drug-resistant hypertensive state, ${ }^{1,2,5,6}$ and 3) adherence to antihypertensive drug treatment may be particularly poor. ${ }^{4-6}$

The therapeutic approach aimed at lowering elevated blood pressure values in obese hypertensive patients includes both nonpharmacological and pharmacological interventions. ${ }^{4,6}$ Nonpharmacological approaches embrace dietary regimens aimed at reducing caloric intake, pharmacological compounds, and/or surgical interventions and are capable of interfering at different levels with energy food consumption as well as metabolism. In general terms, the blood pressure lowering effects of nonpharmacological interventions display a large interindividual variability and are strictly dependent on the amount of body weight and body fat lost. ${ }^{4,6}$ More importantly, some of these lifestyle interventions are accompanied by the so-called weight-cycling phenomenon, ie, the tendency of the body weight (and in part also of the blood pressure) values to return within months to the preintervention values, with an adverse impact on patients' health and prognosis. ${ }^{8}$ Information on the blood pressure lowering effects of weight loss drugs is more limited, however, because some drugs used have been shown to exert direct pressor action (sibutramine), cause untoward effects (rimonabant), or are still in Phase II investigations (topiramate, liraglutide). ${ }^{4}$ More conclusive are the results provided for bariatric surgery, with the report of a consistent blood pressure reduction associated with the improvement of the metabolic profile at the short-term (1 year) and long-term (11 years) follow-up. ${ }^{4}$

As far as antihypertensive drug treatment is concerned, no indication on the use of preferred antihypertensive drugs in the therapeutic approach of obesity-related hypertension is provided by current hypertension guidelines, which base their recommendations mainly on the evidence provided by specific clinical trials. This is because the clinical trials aimed at exploring the effects of given antihypertensive therapeutic intervention on hard and surrogate end points are lacking in obese hypertensive patients. ${ }^{4,6}$ Some information derived from the analysis of the results obtained in subgroups of patients with hypertension and obesity enrolled in different clinical trials is however available and can be summarized as follows. 
First, it appears to be extremely difficult to achieve blood pressure control in obese hypertensive patients by employing, even at full dosage, a single antihypertensive drug., ${ }^{4,6}$ This is because the multiple pathophysiological mechanisms involved in blood pressure elevation in obese patients make this therapeutic goal difficult to be achieved by a single drug whose mechanism of action is in most cases a single one. ${ }^{4-6}$ This is the case for diuretics, dihydropyridine calcium antagonists, ACE inhibitors, and angiotensin II receptor blockers. ${ }^{4,6}$ This is also the case for a centrally acting sympatholytic drug such as moxonidine, despite the aforementioned evidence that the obese hypertensive state has a pronounced sympathetic overdrive as pathophysiological hallmark., ${ }^{4,6}$ This is finally the case for $\beta$-blocking agents, which reduce elevated blood pressure values in obese patients, although triggering adverse metabolic effects, such as a reduction in insulin sensitivity (and thus a worsening of the insulin resistance state) and an increase in plasma glucose levels. These adverse metabolic effects, however, are not detectable with new $\beta$-blocking agents exerting vasodilating properties. ${ }^{4-6}$

Second, combination drug treatment represents a recommended therapeutic option in obese hypertensives for treatment aimed at obtaining a stable blood pressure control. ${ }^{4-6}$ The information, however, is scanty and not always based on results of clinical studies with a solid experimental design. An exception is represented by the data collected in the Avoiding cardiovascular events through COMbination therapy in Patients Living with Systolic Hypertension (ACCOMPLISH) trial, which followed a double-blind design and evaluated the effects of two combination drug treatments - amlodipine/benazepril or hydrochlorothiazide/ benazepril - on blood pressure values and cardiovascular events as well. ${ }^{9}$ The peculiar feature of the study was the inclusion in the trial population of a noticeable number of hypertensive patients with a body mass index $>30 \mathrm{~kg} / \mathrm{m}^{2}$ (more than 5,000 on a total population of about 11,000). In this specific group, a data subanalysis was carried out. ${ }^{10}$ Both the drug combinations allowed the achievement of blood pressure control in about two-thirds of the patients enrolled, the blood pressure lowering effects of the thiazide/ACE inhibitor combination treatment being similar to that documented for the calcium antagonist/ACE inhibitor. However, when the data were analyzed examining the effects of the two types of drug combination on cardiovascular outcomes (specifically cardiovascular deaths, nonfatal myocardial infarctions, or strokes), it was found that in normoweight and in overweight patients, benazepril/amlodipine guaranteed cardiovascular protection greater than the hydrochlorothiazide/benazepril.
Surprisingly, this was not the case in obese hypertensive patients in whom the diuretic/ACE inhibitor combination appeared to be associated with an event rate lower than that seen for the other drug combination tested in the study. Although the results of the trial related to the cardioprotective effects of the two combination treatments are difficult to interpret, ACCOMPLISH data provide evidence that the therapeutic approach based on the association of an ACE inhibitor, a thiazide diuretic, or a calcium antagonist is capable of guaranteeing the achievement of a satisfactory blood pressure control in a consistent group of obese hypertensive patients. Almost superimposable results were obtained in a smaller study, in which, however, the use of 24-hour ambulatory blood pressure monitoring allowed the confirmation of efficacy of the combination losartan/hydrochlorothiazide throughout the 24-hour period, and particularly during the nighttime in obese hypertensive patients aged more than 65 years. ${ }^{11}$ Similar effects were obtained in other studies in which the combination was based on angiotensin II receptor blockers and diuretics or $\beta$-blockers and diuretics. ${ }^{12-15}$ Finally, results of two recent meta-analyses, one based on the data collected in three clinical trials recruiting more than 12,000 patients and the other in seven controlled clinical trials recruiting about 6,000 hypertensive patients, have provided evidence that the combination valsartan/amlodipine or aliskiren/amlodipine may guarantee a blood pressure control of similar magnitude both in nonobese and in obese patients. ${ }^{16,17}$

Obesity-related hypertension is quite frequently associated with an increased incidence of the so-called nondipping phenomenon with the loss of the physiological blood pressure fall during nighttime sleep, and thus with the occurrence of alterations in circadian 24-hour blood pressure profile. ${ }^{18}$ Such alterations, which also include an increase in pulse pressure, ${ }^{18}$ in the magnitude of the morning blood pressure surge, ${ }^{18}$ and in 24-hour blood pressure variability ${ }^{18,19}$ are detected in the obese state, particularly when this condition is complicated by the sleep apnea syndrome. ${ }^{19,20}$ The aforementioned blood pressure abnormalities represent key therapeutic targets because of the evidence that their presence increases the cardiovascular risk profile of a given patient as well as the incidence of end-organ damage. ${ }^{21,22}$ The effects of antihypertensive drug combination treatment on these different targets have been investigated in few studies specifically performed in obese hypertensive patients; however, as mentioned, the combination losartan/hydrochlorothiazide has been shown to restore the blood pressure reduction occurring during nighttime. ${ }^{11}$ Similar effects have been reported for the telmisartan/hydrochlorothiazide as well as for the 
fosinopril/hydrochlorothiazide combination, ${ }^{23,24}$ while pulse pressure values have been shown to be significantly reduced by valsartan/hydrochlorothiazide. ${ }^{13,25}$ In general terms, the results of these studies suggest that the drug combination more effective in correcting the abnormalities of the blood pressure profile described in the obese hypertensives should necessarily include a drug acting on the renin-angiotensin system.

\section{Basic pharmacology profile of enalapril/lercanidipine combination}

In discussing the main pharmacological features of the drug combination, ${ }^{26}$ it should be worth mentioning that the presence of an obese state may affect the main pharmacological properties of a given drug, particularly the so-called volume of distribution of the compound, ie, the drug location into different interstitial and intracellular fluids after absorption or direct administration. ${ }^{27}$ Factors affecting this variable include the physical volume of the organism, the plasma protein binding, and the removal rate, which in turn depends on tissue blood flow. This latter parameter, which is reduced in the obese state particularly when high blood pressure is present, may affect the pharmacokinetic profile of a drug, in conjunction with the drug clearance, which depends on the hepatic and renal metabolic rate. ${ }^{27}$

\section{Pharmacological profile}

Lercanidipine is a calcium channel blocker of the dihydropiridine group that displays specific binding activities for the receptor subunit a1 of the calcium channels type $\mathrm{L}$, thereby inhibiting the influx of calcium into the cell. ${ }^{28,29}$ Similar to other compounds of the same pharmacological class, the blood pressure lowering effects of lercanidipine mainly depend on the enantiomer " $S$ ". The drug is highly liphophilic, allowing the achievement of a rapid diffusion and a prolonged durability within the phospholipidic layer of the smooth muscle cells of the arterial wall. This property allows the compound to produce a smooth and prolonged antihypertensive effect (which is dose dependent and based on a reduction in total peripheral vascular resistance); the value of its trough-to-peak ratio at the daily dosage of $10 \mathrm{mg}$ being positioned in the high-scale range $(0.84){ }^{20,21}$ It also allows lercanidipine to guarantee a favorable side effect profile as compared to classic calcium channel blockers. ${ }^{28,29}$ Along with the antihypertensive effects, lercanidipine has been shown to exert antiatherogenic properties, documented by the ability of the drug to inhibit smooth muscle cell proliferation and migration as well macrophages esterification of cholesterol in animal models. ${ }^{28,29}$ The recommended daily dosage is $10-20 \mathrm{mg}$.

Enalapril is a drug acting on the renin-angiotensinaldosterone axis by inhibiting the activity of the angiotensinconverting enzyme chimase II, which is responsible for the synthesis of angiotensin II from angiotensin I. ${ }^{30}$ Enalapril is a prodrug that is converted to the active formulation enalaprilat after hepatic hydrolysis. The drug at a daily dosage of $20 \mathrm{mg}$ displays a peak effect 6-8 hours following oral administration, and its blood pressure lowering effects, which are dose dependent, appear to be maintained for the 24-hour period. ${ }^{30}$ Similar to lercanidipine, the drug exerts its action by reducing total peripheral vascular resistance, and in addition to its antihypertensive effects, it may favorably interfere with renal function and with some initial developing steps of the atherogenic process, such as platelets activation. ${ }^{30}$ The recommended daily dosage is $10-20 \mathrm{mg}$.

\section{Pharmacokinetic profile}

The pharmacokinetics properties of lercanidipine have been investigated in healthy male volunteers and in patients with hypertension or renal insufficiency and failure (at different ages). ${ }^{28}$ The drug, after oral administration, is quickly absorbed at gastrointestinal level and undergoes first-pass metabolism by the enteric isoenzyme cytochrome P450 (CYP) 3A4. ${ }^{28}$ Given the evidence that the absorption of lercanidipine from the gastrointestinal tract is fourfold after a high-fat meal, the drug should be administered prior to meals. The absolute bioavailability of lercanidipine after a single oral administration of the drug at a dosage of $10 \mathrm{mg}$ is about $30 \%$, while the peak plasma concentrations of radioactivity in human studies employing the radiolabeled drug occurred 1 hour after dose administration. ${ }^{28}$ Lercanidipine is metabolized to inactive metabolites, and about $45 \%$ is excreted in urine. Renal insufficiency and failure may affect the pharmacokinetic profile of the drug. Given the results of in vitro studies, the drug should not be coadministered with other substances inhibiting enteric isoenzyme CYP. ${ }^{28}$

The pharmacokinetic data collected for enalapril are based on studies carried out in healthy male volunteers and in patients with hypertension or renal insufficiency and failure at different ages. ${ }^{30}$ The drug, after oral administration, is absorbed at the gastrointestinal level and is metabolized at hepatic level to the active form enalaprilat. The drug absorption is not affected by meals. ${ }^{30}$ The absolute bioavailability of enalapril after a single oral administration of the drug at the dosage of $20 \mathrm{mg}$ is about $40 \%$, while the peak plasma concentrations of radioactivity in human studies employing the 
radiolabeled drug occurred 1 hour after dose administration for enalapril and 3-4 hours for enalaprilat. ${ }^{30}$ Enalapril crosses the placental barrier, a finding that contraindicates its use alone or combined with other drugs (including lercanidipine) in women with documented or suspected pregnancy. ${ }^{30}$ The drug is metabolized to inactive metabolites and about $60 \%$ is excreted in urine. Both renal insufficiency and failure may affect the pharmacokinetic profile of the drug, this being the case also for congestive heart failure. ${ }^{30}$

A study carried out in hypertensive patients has documented some pharmacokinetic features of the fixed combination, ${ }^{26}$ such as the value of the peak plasma concentrations of lercanidipine, enalapril, and enalaprilat (respectively 3.3, 91 , and $53 \mu \mathrm{g} / \mathrm{L}$ ) obtained with a single daily oral dose of the combo drug treatment lercanidipine $10 \mathrm{mg}$ plus enalapril $20 \mathrm{mg} .{ }^{26}$ The bioequivalence acceptance range was fixed between 0.80 and $1.25 .^{26}$

\section{Efficacy studies in obese hypertensive patients}

According to the recommendations jointly issued by the European Society of Hypertension and the European Society of Cardiology, an antihypertensive drug combination should fulfill a number of requirements and possess desirable features (Table 1). ${ }^{6}$ Traditionally, combination drug treatment has been evaluated taking into account a number of effects, such as the rate of blood pressure (particularly systolic) control, the efficacy in comparison of single monotherapies and other drug combinations, the tolerability, the side effects profile, and the impact on treatment compliance. ${ }^{6}$ Guidelines have also indicated the preferred drug combinations to be adopted in the single patient, taking into account several variables including the cardiovascular risk profile of the individual patient. ${ }^{6}$ The combination drug treatment based on a calcium channel blocker and an ACE inhibitor represents one of the most effective therapeutic options, which has been successfully tested in a large number of clinical trials. ${ }^{6}$ The results of these studies have shown the superiority of this combination

Table I Requirements and desirable features of antihypertensive drug combination treatment

- Greater hemodynamic/blood pressure lowering effects than single agents

- Efficacy in broad range of subject types

- Tolerability profile at least comparable to single agents

- Properties capable to enhance compliance to treatment

- Effects on mechanisms of blood pressure control

- Evidence for long-term benefits

- Potential utility across cardiovascular continuum treatment in terms of 1) antihypertensive efficacy when compared to a $\beta$-blocker/diuretic combination, ${ }^{6} 2$ ) fatal and nonfatal cardiovascular events reduction when compared to an ACE inhibitor/diuretic combination, and 3) the absence of dysmetabolic effects when compared to an angiotensin II receptor antagonist/diuretic combination. ${ }^{6}$

\section{Comparative studies with placebo or monotherapy}

The blood pressure lowering effects of lercanidipine/enalapril in obese hypertensive patients have been evaluated in a number of recently performed studies, comparing its efficacy versus placebo or monotherapy based on one or the other drug of the combination.

In a large-scale investigation, the efficacy and safety of lercanidipine and enalapril in patients with essential hypertension (FELT) study, carried out in 100 centers located in seven European countries according to a randomized, doubleblind, placebo-controlled design, Mancia et $\mathrm{al}^{31}$ assessed the office and home blood pressure lowering effects of different daily doses of the combination (lercanidipine/enalapril 10/10, $10 / 20,20 / 10$, and 20/20 mg/d). The study enrolled more than 1,000 hypertensive patients, with an average body mass index close to $30 \mathrm{~kg} / \mathrm{m}^{2}$. About $40 \%$ of the enrolled patients displayed a body mass index greater than $30 \mathrm{~kg} / \mathrm{m}^{2}$, and this was affected by an obese state. Office systolic and diastolic blood pressure values were significantly reduced by all the four combination regimens, the greatest effects being shown by the highest daily dosage $20 \mathrm{mg} / 20 \mathrm{mg}$, which showed the highest responder and normalization rate. It is worth mentioning that these effects were consistent even when home blood pressure, which is virtually devoid of any significant placebo effect, replaced clinic blood pressure measurements. These results have been recently strengthened by an additional analysis of the database of the FELT study, comparing the effects of the drug combination treatment to single monotherapies on home blood pressure values. ${ }^{32}$ In general, the blood pressure lowering effects of the lercanidipine/enalapril combination were shown to be homogeneous in the whole study population, including the consistent group of obese patients, thereby indicating the efficacy of lercanidipine/ enalapril in obesity-related hypertension.

The results of these studies confirmed previous findings collected in two large-scale multicenter prospective studies carried out in large cohorts of patients who are overweight or overtly obese and have hypertension. In the first study, data on the efficacy of the fixed combination lercanidipine $10 \mathrm{mg} / \mathrm{enal}-$ april $20 \mathrm{mg}$ were evaluated in more than 8,000 hypertensive 
patients followed on average for a 3-month period. ${ }^{33}$ Seventy percent of the study population showed concomitant diseases, including about one-third of the recruited patients being obese. Although no separate analysis of the results was made in the obese or in the normoweight subgroups, the data confirmed the marked blood pressure lowering effects of the drug combination in the study population, independent of the presence or absence of obesity. On average, the clinic systolic and diastolic blood pressure reduction detected at the end of the study period amounted to 28.4 and $13.5 \mathrm{mmHg}$, respectively, with target blood pressure below 140/90 $\mathrm{mmHg}$ being achieved in $79 \%$ of the patients. Similar figures of efficacy were reported in another study that recruited patients with an average body mass index amounting to $29 \mathrm{~kg} / \mathrm{m}^{2}$, with about $10 \%$ of them suffering from an obese state frequently complicated by metabolic alterations. ${ }^{34}$ The unique feature of this investigation was the assessment not only of clinic but also of 24 hour and home blood pressure, allowing the evaluation of the efficacy of the drug combination treatment in daily life conditions as well. Ambulatory blood pressure values were significantly decreased by the combination drug regimen throughout the 24-hour period, and this was accompanied by a significant reduction in blood pressure variability index. ${ }^{34}$ This suggests that the lercanidipine/enalapril combination allows to exert protective cardiovascular effects not only by reducing the absolute blood pressure load to the heart but also by the spontaneous oscillations of this hemodynamic variable occurring during the daytime and the nighttime period. Given the well-defined adverse impact of blood pressure variability for cardiovascular prognosis, ${ }^{35}$ this latter result may have favorable implications for the treatment-induced reduction of the patient's risk profile.

\section{Comparative studies with other drug combinations}

Four studies compared the efficacy of lercanidipine/enalapril with other drug combination regimens in obese or overweight hypertensive patients. ${ }^{7,36-38}$ Three studies compared the efficacy of lercanidipine $10 \mathrm{mg} /$ enalapril $20 \mathrm{mg}$ to enalapril $20 \mathrm{mg}$ /hydrochlorothiazide $12.5 \mathrm{mg}$ combination in different populations of hypertensive patients. ${ }^{7,36,37}$ The first study, ${ }^{36}$ carried out in diabetic hypertensive patients with a body mass index $>30 \mathrm{~kg} / \mathrm{m}^{2}$ uncontrolled by enalapril treatment, has shown a greater efficacy in terms of clinic blood pressure reduction of lercanidipine $10 \mathrm{mg}$ when compared to hydrochlorothiazide $12.5 \mathrm{mg}$ as add-on to the ACE inhibitor treatment based on enalapril $20 \mathrm{mg}$. Indeed, following a 20-week follow-up, the percentage of responders, defined as diastolic blood pressure decrease $\geq 10 \mathrm{mmHg}$ or as achievement of blood pressure values $\leq 130 / 85 \mathrm{mmHg}$, was greater in the group under the former than under the latter combination treatment. In another study, performed in overweight hypertensives, lercanidipine $20 \mathrm{mg}$ /enalapril $20 \mathrm{mg}$ treatment produced a systolic and diastolic blood pressure reduction slightly greater than the lercanidipine $20 \mathrm{mg} /$ hydrochlorothiazide $25 \mathrm{mg}$ combination. ${ }^{7}$ More importantly, the abnormalities in the retinal microcirculation (increased media-to-lumen ratio of the retinal arterioles), as well as the abnormal increase in arterial stiffness, derived from central blood pressure measurement, were improved only by lercanidipine/enalapril. ${ }^{7}$ It thus appears that this drug combination exerts cardiovascular effects additive to the blood pressure reduction by favoring a regression of the vascular structural alterations detectable in hypertension at the level of the macrocirculation and microcirculation as well. These effects, also known as "ancillary properties" of a given antihypertensive drug or drug combination, are likely to depend on the antioxidative and anti-inflammatory action of lercanidipine at the endothelial level. Similarly, a recent study carried out in hypertensive patients with central obesity and metabolic syndrome has shown greater effects of the lercanidipine 10-20 mg/enalapril $20 \mathrm{mg}$ combination as compared to lercanidipine $10-20 \mathrm{mg} /$ hydrochlorothiazide 12.5-25 mg on augmentation index, ie, a sensitive marker of wave reflection and arterial stiffness derived from the blood pressure waveform. ${ }^{37}$

Finally, our group has recently completed a double-blind crossover study in hypertensive patients with central obesity, aimed at comparing the hemodynamic as well as metabolic effects of lercanidipine 10-20 mg/enalapril $20 \mathrm{mg}$ versus felodipine 5-10 mg/enalapril $20 \mathrm{mg}$ in this very high-risk population. ${ }^{38}$ The study was based on both clinic and ambulatory blood pressure measurement and included, as a unique feature, the assessment of efferent postganglionic muscle sympathetic nerve traffic in the peroneal nerve via the microneurographic technique. ${ }^{2}$ The approach indeed represents the gold standard method to assess human cardiovascular adrenergic drive in a sensitive, reproducible, and direct manner. ${ }^{2}$ The results show that the two drug combinations provide similar sphygmomanometric and 24-hour ambulatory blood pressure reductions. However, when the sympathetic and the metabolic data were examined, it became clear that compared to enalapril-felodipine, enalapril-lercanidipine was almost entirely devoid of any major stimulating 
effect on cardiac and peripheral cardiovascular drive. This was documented by the lack of any significant heart rate and sympathetic nerve traffic increase during lercanidipine/enalapril treatment, which was different from what was observed during the felodipine/enalapril administration. Interestingly, the absence of sympathoexcitatory effects displayed by the lercanidipine/enalapril combination was coupled with a significant improvement in insulin sensitivity, which was different from what was detected during felodipine/enalapril treatment. ${ }^{38}$ This finding confirms the close pathophysiological dependence of insulin resistance on sympathetic activation. It also supports the notion that a pharmacological modulation of adrenergic cardiovascular drive may result in favorable metabolic effects. ${ }^{2}$ It finally represents a result of key clinical relevance, taking into account that 1) obese patients (particularly when hypertensive) almost invariably display an insulin-resistance state and 2) in obesity-related hypertension, insulin resistance is involved in the development and progression of the vascular alterations (particularly vascular fibrosis and arterial stiffness) and is associated with an increased incidence of fatal and nonfatal cardiovascular events. ${ }^{2,39}$ The results of this study are strengthened by the finding that, compared to other combinations, such as lercanidipine/hydrochlorothiazide, lercanidipine/enalapril is able to correct the alterations of insulin signaling process occurring in the skeletal muscle. This peculiar property is documented by the finding that during a 6-month treatment with this drug combination, an increased expression of insulin receptor glucose transporter 4 and an increased activation of the Chinese p70-S6K 1 could be detected..$^{40}$ However, assessment of the effects of lercanidipine/enalapril combination on the metabolic profile of the obese or overweight hypertensive patient is not limited to insulin resistance. Indeed, data collected in the aforementioned study as well as in other studies provided evidence that this drug combination significantly increases plasma high-density lipoprotein cholesterol values, concomitantly reducing circulating plasma triglycerides as well as plasma lipoprotein-a levels. ${ }^{7,36-38,41}$

\section{Safety and tolerability}

The studies analyzed in this review also investigated the occurrence of side effects as well as the tolerability profile of the lercanidipine/enalapril combination in obese hypertensive patients. In general, the results are completely in line with those reported in other studies carried out in other hypertensive populations uncomplicated by obesity. ${ }^{42}$ The largest study already discussed in this review (more than 8,000 patients enrolled) showed that the tolerability profile of this drug combination was satisfactory, with adverse side effects being recorded in less than $2 \%$ of the recruited patients. ${ }^{33}$ Cough and peripheral edema, ie, the classic side effects of an ACE inhibitor and a calcium channel blocker, respectively, were reported only in $0.5 \%$ and $0.1 \%$ of the patients, while flushing and dizziness were reported in $0.2 \%$. In the same study, an assessment of the treatment tolerability profile showed that $69.0 \%$ of the patients referred to it as "very good", $28.4 \%$ as "good", $1.4 \%$ as "satisfactory", and $1.2 \%$ as "poor". ${ }^{33}$ Since good tolerability is frequently associated with good patient compliance to treatment, the adherence of the patients to the lercanidipine/enalapril combination in obese hypertensive patients was regarded as excellent, as also reported in the studies employing the same combination in uncomplicated hypertensive patients. $^{42}$

\section{Potential limitations of the drug combination}

Similar to other drug combinations used in hypertension treatment, lercanidipine/enalapril displays a number of potential limitations. One of them is represented by the fact that it makes use, as ACE inihibitor, of enalapril, ie, a drug that not infrequently has been shown to fail to exert a full therapeutic coverage of the 24-hour period..$^{30}$ However, the data collected in the previously mentioned FELT study as well as those reported in the database of 854 patients do not provide evidence on any lack of 24-hour blood pressure control with the use of lercanidipine/enalapril combination. ${ }^{31,32}$ This suggests that in the combination treatment, lercanidipine may counterbalance this specific enalapril limitation. The second limitation is that lercanidipine, although showing favorable effects on different measures of target organ damage, has never been tested in clinical studies aimed at determining the drug effects on hard end points. ${ }^{28,29}$ However, the lack of such information, in the case of the drug combination with enalapril, is counterbalanced by the data collected in outcome clinical trials making use of enalapril during the past 30-35 years. ${ }^{30}$

\section{Conclusion}

According to guidelines and consensus documents issued by major scientific societies, blood pressure control in treated obese hypertensive patients represents a major goal of the therapeutic intervention, which can be achieved in the majority of cases with the use of combination drug treatment. The drug combination lercanidipine/enalapril represents a therapeutic approach, which can be favorably 
used in the treatment of this condition. The results of the studies reviewed in this paper emphasize the efficacy, safety, and tolerability profile of this drug combination. In addition to its blood pressure lowering action, lercanidipine/enalapril may exert favorable effects on a number of variables, such as the sympathetic abnormalities, the endothelial dysfunction, as well as the metabolic alterations, which represent the hallmarks of the obese hypertensive state.

\section{Disclosure}

The author reports no conflicts of interest in this work.

\section{References}

1. Hall JE, do Carmo JM, da Silva AA, Wang Z, Hall ME. Obesity-induced hypertension: interaction of neurohumoral and renal mechanisms. Circ Res. 2015;116(6):991-1006.

2. Grassi G, Mark A, Esler M. The sympathetic nervous system alterations in human hypertension. Circ Res. 2015;116(6):976-990.

3. O'Neil S, O'Driscoll L. Metabolic syndrome: a closer look at the growing epidemic and its associated pathologies. Obes Rev. 2015;16(1): $1-12$.

4. Jordan J, Yumuk V, Schlaich M, et al. Joint statement of the European Association for the Study of Obesity and the European Society of Hypertension: obesity and difficult to treat arterial hypertension. J Hypertens. 2012;30(6):1047-1055.

5. Jordan J, Nilsson PM, Kotsis V, et al. Joint scientific statement of the European Association for the Study of Obesity and the European Society of Hypertension: obesity and early vascular aging. J Hypertens. 2015;33(3):425-434.

6. Mancia G, Fagard R, Narkiewicz K, et al. Task Force Members. 2013 ESH/ESC Guidelines for the management of arterial hypertension: the Task Force for the management of arterial hypertension of the European Society of Hypertension (ESH) and of the European Society of Cardiology (ESC). J Hypertens. 2013;31(7):1281-1357.

7. De Ciuceis C, Salvetti M, Rossini C, et al. Effect of antihypertensive treatment on microvascular structure, central blood pressure and oxidative stress in patients with mild essential hypertension. $J$ Hypertens. 2014;22(3):565-574.

8. Mehta T, Smith DL, Muhammad J, Casazza K. Impact of weight cycling on risk of morbidity and mortality. Obes Rev. 2014;15(11):870-881.

9. Jamerson K, Weber MA, Bakris GL, et al; for the ACCOMPLISH Trial Investigators. Benazepril plus amlodipine or hydrochlorothiazide for hypertension in high-risk patients. N Engl J Med. 2008;359(23): $2417-2428$.

10. Weber, MA, Jamerson K, Bakris GL, et al. Effects of body size and hypertension treatments on cardiovascular event rates: subanalysis of the ACCOMPLISH randomized controlled trial. Lancet. 2013;381(9866):537-545.

11. Ibuki C, Seino Y, Otsuka T, Mizuno K. The fixed-dose combination of losartan/hydrochlorothiazide elicits potent blood pressure lowering during nightime in obese hypertensive patient. J Clin Med Res. 2014;6(1):8-16.

12. Ofili EO, Zappe DH, Purkayastha D, Samuel R, Sowers JR. Antihypertensive and metabolic effects of angiotensin receptor blocker/diuretic combination therapy in obese, hypertensive African American and white patients. Am J Ther. 2013;20(1):2-12.

13. Raij L, Egan BM, Zappe DH, Purkayastha D, Samuel R, Sowers JR. Office and ambulatory blood pressure-lowering effects of combination valsartan/hydrochlorothiazide vs. hydrochlorothiazide-based therapy in obese, hypertensive patients. J Clin Hypertens (Greenwich). 2011;13(10):731-738.
14. Neutel JM. A comparison of the efficacy and safety of irbesartan/ hydrochlorothiazide combination therapy with irbesartan monotherapy in the treatment of moderate or severe hypertension in diabetic and obese hypertensive patients: a post-hoc analysis review. Postgrad Med. 2011;123(4):126-134.

15. Kelly AS, Gonzalez-Campoy JM, Rudser KD, et al. Carvedilol-lisinopril combination therapy and endothelial function in obese individuals with hypertension. J Clin Hypertens (Greenwich). 2012;14(2):85-91.

16. Liu Y, Chen K, Kou X, Han Y, Zhou L, Zeng C. Aliskiren and amlodipine in the management of essential hypertension: meta-analysis of randomized controlled trials. PLoS One. 2013;8(7):e70111.

17. Eckert S, Freytag SB, Müller A, Klebs SH. Meta-analysis of three observational studies of amlodipine/valsartan in hypertensive patients with additional risk factors. Blood Press. 2013;22(Suppl 1):11-21.

18. Kotsis V, Stabouli S, Bouldin M, Low A, Toumanidis S, Zakapoulos N. Impact of obesity on 24-hour ambulatory blood pressure and hypertension. Hypertension. 2005;45(4):602-607.

19. Noda A, Okada T, Kayashi H, Yasuma F, Yokota M. 24-hour ambulatory blood pressure variability in obstructive sleep apnea syndrome. Chest. 1993;103(5):1343-1347.

20. Nabe B, Lies A, Pankow W, Kohl FV, Lohmann FW. Determinants of circadian blood pressure rhythm and blood pressure variability in obstructive sleep apnoea. J Sleep Res. 1995;4(S1):97-101.

21. Grassi G, Bombelli M, Seravalle G, Brambilla G, Dell'Oro R, Mancia G. Role of ambulatory blood pressure monitoring in resistant hypertension. Curr Hypertens Rep. 2013;15(3)232-237.

22. Mancia G, Grassi G. Mechanisms and clinical implications of blood pressure variability. J Cardiovasc Pharmacol. 2000;35(Suppl 4):S15-S19.

23. Fogari R, Mugellini A, Zoppi A, et al. Effect of telmisartan/ hydrochlorothiazide combination on ambulatory blood pressure and cognitive function in elderly hypertensive patients $J$ Hum Hypertens. 2006;20(3):177-185.

24. Guthrie R, Reggi DR, Plesher MM, Saini RK, Battikha JP. Efficacy and safety of fosinopril/hydrochlorothiazide combinations on ambulatory blood pressure profiles in hypertension. Fosinopril/Hydrochlorothiazide Investigators. Am J Hypertens. 1996;9(4):306-311.

25. Ruilope LM, Malacco E, Khder Y, kandra A, Bonner G, Heintz D. Efficacy and tolerability of combination therapy with valsartan plus hydrochlorothiazide compared with amlodipine monotherapy in hypertensive patients with other cardiovascular risk factors: the VAST study. Clin Ther. 2005;27(5):578-587.

26. Recordati SpA. Lercanidipine-enalapril interaction (PK 0031) [data on file]. Milan, Italy: Recordati SpA; 2004.

27. Pai MP. Drug dosing based on weight and body surface area: mathematical assumptions and limitations in obese adults. Pharmacotherapy. 2012;32(9):856-868.

28. McClellan KJ, Jarvis B. Lercanidipine: a review of its use in hypertension. Drugs. 2003;60(5):1123-1140.

29. Borghi C. Lercanidipine in hypertension. Vasc Health Risk Manag. 2005;1(3):173-182.

30. Todd PA, Goa KL. Enalapril. A reappraisal of its pharmacology and therapeutic use in hypertension. Drugs.1992;43(3);346-381.

31. Mancia G, Coca A, Chazova I, et al; on behalf of the FELT Investigators. Effects on office and home blood pressure of the lercanidipine-enalapril combination in patients with stage 2 hypertension: a European randomized, controlled clinical trial. J Hypertens. 2014;32(8):1700-1707.

32. Mancia G, Omboni S, Chazova I, et al. Effects of the lercanidipineenalapril combination vs the corresponding monotherapies on home blood pressure in hypertension. Evidence from a large database. J Hypertens. 2016;34(1):139-148.

33. Rump LC. Efficacy and tolerability of the fixed lercanidipine-enalapril combination in the treatment of patients with essential hypertension. Arzneimittelforschung. 2010;60(3)124-130.

34. Scholze J, Bramlage P, Trenkwalder P, Kreutz R. Efficacy and safety of a fixed-dose combination of lercanidipine and enalapril in daily practice. A comparison of office, self-measured and ambulatory blood pressure. Expert Opin Pharmacother. 2011;12(17):1-9. 
35. Mancia G. Short- and long-term blood pressure variability: present and future. Hypertension. 2012;60(2):512-517.

36. Agrawal R, Marx A, Haller H. Efficacy and safety of lercanidipine versus hydrochlorothiazide as add-on to enalapril in diabetic populations with uncontrolled hypertension. J Hypertens. 2006;24(1):185-192.

37. Ghiadoni L. Bruno MR, Cartoni G, et al. Combination therapy with lercanidipine and enalapril reduced central blood pressure augmentation in hypertensive patients with metabolic syndrome. Vascul Pharmacol. June 10, 2015. Available from: http://dx.doi.org/10.1016/ j.vph.2015.06.004. Accessed March 19, 2016.

38. Seravalle G, Brambilla G, Prata Pizzalla D, et al. Differential effects of enalapril-felodipine vs enalapril-lercanidipine combination drug treatment on sympathetic nerve traffic and metabolic profile in obesityrelated hypertension. J Am Soc Hypertens. Epub 2016 Jan 11.
39. Sethi S, Rivera O, Oliveros R, Chilton R. Aortic stiffness: pathophysiology, clinical implications, and approach to treatment. Integr Blood Press Control. 2014;7(5):29-34.

40. De Ciuceis C, Flati V, Rossini C, et al. Effect of antihypertensive treatment on insulin signalling in lympho-monocytes of essential hypertensive patients: a pilot study. Blood Press. 2014;23(6):330-338.

41. Derosa G, Bonaventura A, Romano D, et al. Effects of enalapril lercanidipine combination on some emerging biomarkers in cardiovascular risk stratification in hypertensive patients. J Clin Pharm Ther 2014;39(3):277-285.

42. Hair PI, Scott LJ, Perry CM. Fixed-dose combination lercanidipineenalapril. Drugs. 2007;67(1):95-106.

\section{Publish your work in this journal}

Integrated Blood Pressure Control is an international, peer-reviewed open-access journal focusing on the integrated approach to managing hypertension and risk reduction. Treating the patient and comorbidities together with diet and lifestyle modification and optimizing healthcare resources through a multidisciplinary team approach constitute key features of the journal. This journal is indexed on American Chemical Society's Chemical Abstracts Service (CAS). The manuscript management system is completely online and includes a very quick and fair peerreview system, which is all easy to use. Visit http://www.dovepress.com/ testimonials.php to read real quotes from published authors.

Submit your manuscript here: http://www.dovepress.com/integrated-blood-pressure-control-journal 